\title{
Correlation between Alteration of Sharp-wave Ripple Coupled Cortical Oscillation and Long-term Memory Deficit in Alzheimer Disease Model Mice
}

\author{
Hyunwoo Yang and Yong Jeong* \\ Department of Bio and Brain Engineering, KI for Health Science and Technology, KAIST, Daejeon 34141, Korea
}

\begin{abstract}
Alzheimer's disease (AD) is the most common cause of dementia, characterized by prominent episodic memory dysfunction. Recent studies have suggested that there is a sequential mechanism in the memory deficit, with long-term ones preceding short-term ones. However, there is lack of explanation for these symptoms. Interaction between the hippocampus and retrosplenial cortex (RSC) during slow-wave sleep (SWS) is a crucial step for successful long-term memory formation. In particular, sharp-wave ripple (SWR) is a principal hippocampus oscillation that coordinates with RSC activity. To determine the relationship between memory dysfunction and SWR-related oscillation changes in AD, we implanted local field potential electrodes in the hippocampus and RSC of AD model mice (APP/PS1). We found that the SWR-coupled ripple wave increased in the RSC, while the amplitude of the SWR was preserved. In addition, the corresponding delta power in hippocampus and RSC was elevated, together with altered delta synchrony in AD mice. All these findings showed a significant correlation with long-term memory deficits measured in contextual fear conditions. Our study suggests that altered SWR-coupled oscillations are a possible underlying mechanism of episodic memory dysfunction in AD mice.
\end{abstract}

Key words: Alzheimer disease, Episodic memory, Slow-wave sleep, Brain waves

\section{INTRODUCTION}

Alzheimer's disease (AD) is the most common cause of dementia and is characterized by a progressive loss of cognitive function and additional neuropsychiatric symptoms. One of the earliest and most prominent symptoms of $\mathrm{AD}$ is episodic memory dysfunction [1-4]. Although the initial episodic memory deficit is well known, the details of amnesia progression with respect to memory storage process remains uncertain because of the variation in disease status of patients and memory tests [5-7]. Several human studies have suggested that episodic memory disruption in $\mathrm{AD}$ patients is

Submitted November 24, 2021, Revised December 16, 2021,

Accepted December 20,2021

* To whom correspondence should be addressed. TEL: 82-42-350-4324, FAX: 82-42-864-5318 e-mail:yong@kaist.ac.kr due to ineffective consolidation or storage of new information [811]. These studies showed that $\mathrm{AD}$ patients rapidly forget encoded information as the disease progresses. In addition to investigations in humans, studies using AD mice models showed that long-term memory deficits precede short-term memory deficits [12, 13]. These results indicate that there might be a sequential mechanism in the episodic memory dysfunction of $\mathrm{AD}$; however, there is still a lack of explanation for these symptoms.

Appropriate communication between the hippocampus and cortices is critical for proper future use of encoded information. According to previous studies, this interplay is extensively executed during slow wave sleep (SWS), which is characterized by large-amplitude delta waves [14-17]. Neural components related to previous events are reactivated, corresponding synaptic connections are strengthened, and irrelevant ones are globally scaled down during the unique oscillations of SWS [18-20]. Sharp-wave ripple (SWR) is a principal SWS oscillation generated in the hip- 
pocampus. It is a high-frequency $(100 \sim 250 \mathrm{~Hz})$ oscillation that occurs with large-amplitude sharp waves [21]. Sharp-wave ripple leads to hippocampal synaptic plasticity and spreads out and influences interconnected cortical areas [22].

Recent studies showed that the SWR corresponding oscillation is observed in various cortices including prefrontal cortex, anterior cingulate cortex, posterior cingulate cortex together with retrosplenial cortex (RSC) [23-26]. The common feature of these areas is that they are association cortices having reciprocal connectivity with medial temporal lobe. Therefore, SWR correlated oscillation is considered as the critical factor of information transfer from the hippocampus to cortical regions. Among the areas, the RSC has received a lot of attentions recently because of its direct anatomical projection from the dorsal hippocampus CA1 [23, 24]. A previous report also revealed that the SWR coordinated activity in the RSC was larger than that in other brain areas [24]. Modulation of RSC activity or projection between the hippocampus and RSC during SWS is relevant to memory performance, which implies that valid communication between the regions is essential $[27,28]$. Together with SWR, slow oscillation (SO) and spindle events detected in the cortex play an important role in memory consolidation. These oscillations tend to build temporal relationships with each other, indicating mutual information transfer between the hippocampus and cortex [14-17,21].

Although previous findings have proven that cortical interactions with SWR play a crucial role in long-term memory performance, it is still unclear whether or which interaction is responsible for the preceding long-term memory dysfunction in AD. In this study, to determine the possible relationship, we identified the electrophysiological features between the hippocampus and RSC local field potentials (LFPs) during SWS and investigated their relationship with $\mathrm{AD}$ mouse memory performance.

\section{MATERIALS AND METHODS}

\section{Animals}

In this study, we used 12-month-old male APPswe/PSEN1dE9 (AD, APP/PS1) mice with a C57BL/6 and C3H hybrid background. We used 25 wild-type (WT) and 21 transgenic (TG) mice. We housed all the mice under a 12-h light/dark cycle (light phase 09:30 21:30/dark phase 21:30 09:30) and provided them ad libitum access to food and water. All experiments were approved by the Institutional Animal Care and Use Committee of KAIST, and animal care and handling were performed according to ethical guidelines.

\section{Electrode implantation surgery}

We performed surgery under tiletamine-zolazepam/xylazine anesthesia (40 mg/10 mg per kg, i.p.). After the administration of anesthesia, we placed the mice on a stereotaxic frame (KOPF instruments) and implanted tungsten electrodes (\#797550, A-M systems) on the following sites of the right hemisphere: dorsal hippocampus CA1 (AP-1.82 ML+1.25 DV-1.32) and RSC (AP-2.40 $\mathrm{ML}+0.30 \mathrm{DV}-0.80$ ). We implanted a reference electrode (stainless steel screw) within the skull over the cerebellum. A stainless-steel electromyography electrode (\#790700, A-M systems) was located between the neck muscles. Each mouse was single-housed after the surgery and had a recovery time of at least 1 week. We used 18 WT and 14 TG mice (4-day interval group in the contextual fear conditioning test, described below) in the surgery, and excluded five WT and two TG mice because of incorrect recording sites.

\section{$L F P$ recoding}

Before the behavior experiments, each mouse had an LFP recording session at the home cage. The LFP signals were $\times 1,200$ amplified, recorded, and digitized at a $1,600-\mathrm{Hz}$ sampling rate using a digital system (Twin system, GRASS technologies). We simultaneously video-recorded mouse behavior.

\section{SWS classification and sleep oscillation analysis}

We extracted $3 \mathrm{~h}$ of LFP signals during the light phase. We classified sleep state as described in a previous report [24]. First, we manually identified the awake state by the recorded video and filtered electromyography signals (250 500 Hz). Rapid eye movement sleep (REMS) and SWS were distinguished by theta (5 10 $\mathrm{Hz}) /$ delta $(1 \sim 4 \mathrm{~Hz})$ power ratio analyzed using the hippocampus LFP channel. We used the top $25 \%$ ratio as the threshold for dividing the sleep state. We excluded SWS epochs of less than $1 \mathrm{~min}$ from the analysis. We identified SWR, cortical ripple, SO, and spindle within the classified SWS segments. To detect the SWR, the LFP signal from the hippocampus channel was band-pass filtered (ripple band, 100 250 Hz), and the envelope of the filtered signal was calculated using the Hilbert transform. Events above 3 standard deviations of ripple-filtered signal and longer than 15 ms were defined as SWR. We analyzed power spectrograms of LFPs with wideband LFP around the SWR from the hippocampus and RSC. The center of the spectrogram was set to the minimum trough of the ripple oscillation. Each amplitude change in the frequency blocks was z-scored and averaged within the designated frequency band (delta, 1 4 Hz; theta, $5 \sim 10 \mathrm{~Hz}$; beta, 12 30 Hz; gamma, 31 100 Hz; ripple, 100 250 Hz). We adapted the same process to the RSC channel to identify the RSC cortical ripple. Coupling of SWR and RSC ripple was investigated only when RSC 
ripple occurred within $\pm 50 \mathrm{~ms}$ of the SWR trough. We detected SO and spindle as described previously [29]. To detect SO, the LFP signal from the RSC channel was band-pass filtered (SO, 0.5 4 $\mathrm{Hz}$ ) and zero-crossing points were determined. Within intervals between zero-crossing points, we found the interval lasting for 150 500 ms and satisfying the amplitude criterion: maximum peak is higher than $2 \mathrm{SD}$ or higher than $1 \mathrm{SD}$ with a minimum peak lower than $1.5 \mathrm{SD}$. We discovered that upward zero-crossing point $\sim$ maximum peak point minimum peak sequences lasting for $0.15 \sim 0.5 \mathrm{~s}$ and included them in further analysis. To detect spindle, the LFP signal from the RSC channel was band-pass filtered (SPD band, 7 15 Hz), and the envelope of the filtered signal was smoothed with a 100-ms Gaussian window. Events higher than $1.5 \mathrm{SD}$ of filtered signal (and peak value is higher than $2 \mathrm{SD}$ ) for $0.2 \mathrm{~s}$ were identified first. We merged events with intervals less than $0.4 \mathrm{~s}$. Only cases with a final length between 0.4 and $4 \mathrm{~s}$ were included for further analysis. Coupling between the hippocampus SWR and RSC SO was defined as occurrence of SO (maximum peak) within $0.25 \mathrm{~s}$ from the center of SWR. Coupling between the RSC SO and spindle was defined as occurrence of spindle (maximum peak) within $0.1 \sim 1 \mathrm{~s}$ from the end of SO. The two couplings above appeared simultaneously (in terms of SO) and we defined these events as triple case [29-31]. All analyses were based on MATLAB (Mathworks) and filtering process was performed using 'eegfilt' function from the EEGLAB toolbox.

\section{Contextual fear conditioning test}

To identify episodic memory function in mice, we performed a contextual fear conditioning (CFC) test. During the conditioning session, we placed the mouse in a $20 \times 16 \times 20 \mathrm{~cm}$ chamber with a metal grid floor (Med Associates). After 3 min of habituation in the chamber, the mouse received two foot-shocks $(0.7 \mathrm{~mA}, 2 \mathrm{~s})$ at an interval of $1 \mathrm{~min}$ and stayed in the chamber for $1 \mathrm{~min}$ after the final foot shock. To distinguish the short-term and long-term aspects of memory function, we divided the mice into two groups: 2-h interval group (2h ITI, WT=7, TG=7) and 4-day interval group (4d ITI, WT=18, TG=14). We returned both groups to their home cage immediately after the conditioning session and placed them back into the same experimental chamber at different time intervals. During the context recall session, the mice stayed in the chamber for $3 \mathrm{~min}$. We video-recorded all sessions, quantified the freezing behavior manually, and excluded freezing behavior lasting less than 1 s from the analysis.

\section{Open field test}

To measure locomotion and anxiety levels in mice, we conducted an open field test. The test chamber was $40 \times 40 \times 40 \mathrm{~cm}$ and was made of white acryl. The illumination intensity of the chamber was $200 \mathrm{~lx}$. The mouse was placed at the edge of the box and the movement was video-recorded. The total test time was $30 \mathrm{~min}$. We analyzed the distance travelled and time in the center zone (EthoVision XT, Noldus). Only the $4 \mathrm{~d}$ ITI group was used in this test.

\section{Statistical analysis}

All statistical analyses were performed using the GraphPad Prism version 9. We presented data as mean \pm standard error of the mean (SEM). We used the Kolmogorov-Smirnov test as a normality test and analyzed the pass data using Student's t-test. The MannWhitney test was used when normality was not satisfied. We applied Pearson's correlation analysis to determine the significance of linear regression between two parameters.

\section{RESULTS}

\section{Long-term memory deficit in 12-month-old AD mice}

To assess the short-term and long-term memory performance in $\mathrm{AD}$ mice, we performed CFC at distinct time intervals (Fig. 1A). In the $2 \mathrm{~h}$ ITI experiment, WT and TG mice showed similar level of freezing time in after-shock period (Fig. 1B left; WT, 22.24 \pm 3.79 , $\mathrm{n}=7 ; \mathrm{TG}, 16.44 \pm 5.39, \mathrm{n}=7 ; \mathrm{p}=0.396$, Student t-test) and context recall session (Fig. 1B right; WT, 41.21 $\pm 5.61, \mathrm{n}=7$; TG, 45.12 \pm 6.33 , $\mathrm{n}=7 ; \mathrm{p}=0.652$, Student $\mathrm{t}$-test). On the other hand, in the $4 \mathrm{~d}$ ITI experiment, TG mice showed decreased context recall freezing time (Fig. 1C right; WT, 47.09 $\pm 3.76, \mathrm{n}=18$; TG, 28.88 \pm 5.01 , n=14; ${ }^{* *} \mathrm{p}<0.01$, Student's $\mathrm{t}$-test) without unchanging after-shock freezing (Fig. 1C left; WT, 30.78 $\pm 5.52, \mathrm{n}=18$; TG, 20.82 $\pm 6.40, \mathrm{n}=14 ; \mathrm{p}=0.247$, Student's t-test). This result indicates that 12-month-old APP/PS1 mice have intact function in memory encoding and short-term processes, but deficits in relatively longer interval memory. Additionally, we performed an open field test on the $4 \mathrm{~d}$ ITI group mice to verify whether the reduced freezing behavior was due to altered motor function or anxiety in TG mice. There were no differences in the total distance traveled (Fig. 1D left; WT, 8421.76 \pm 575.14 , $\mathrm{n}=18$; TG, $9651.04 \pm 906.88, \mathrm{n}=14 ; \mathrm{p}=0.242$, Student's t-test) and the time spent in the center zone of the open field chamber (Fig. 1D right; $\mathrm{WT}, 126.37 \pm 19.99, \mathrm{n}=18$; TG, $142.09 \pm 22.80, \mathrm{n}=14 ; \mathrm{p}=0.608$, Student's t-test). This result indicates that general motor function and anxiety levels in TG mice are not different from those in WT mice; therefore, the decreased context recall freezing is mainly due to the loss of memory ability.

\section{Increased amplitude of SWR coordinated RSC ripple oscillation in AD mice}

We implanted the LFP electrodes on the hippocampus and 
A

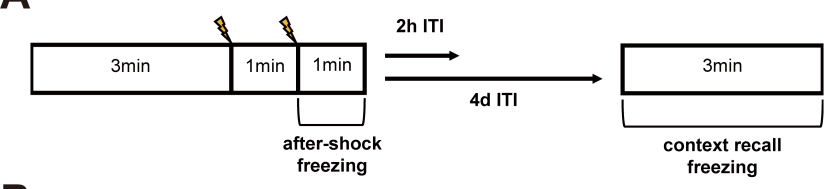

B

after-shock

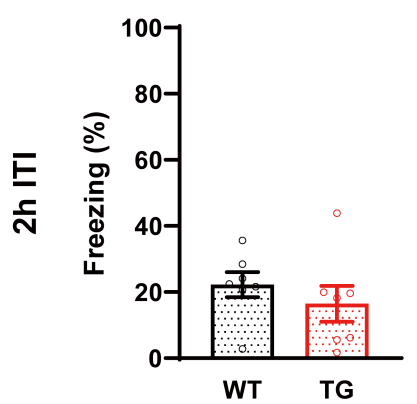

C

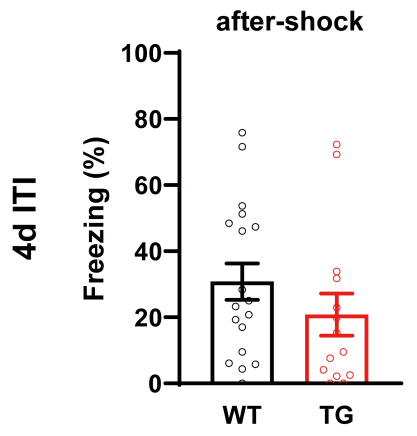

D

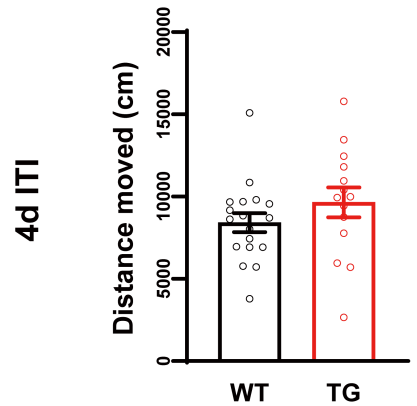

context recall

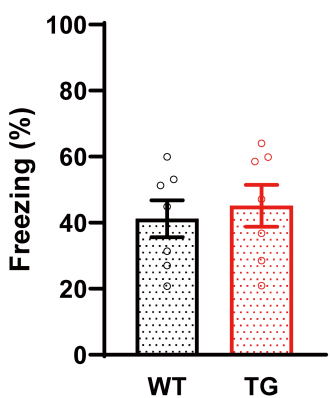

context recall
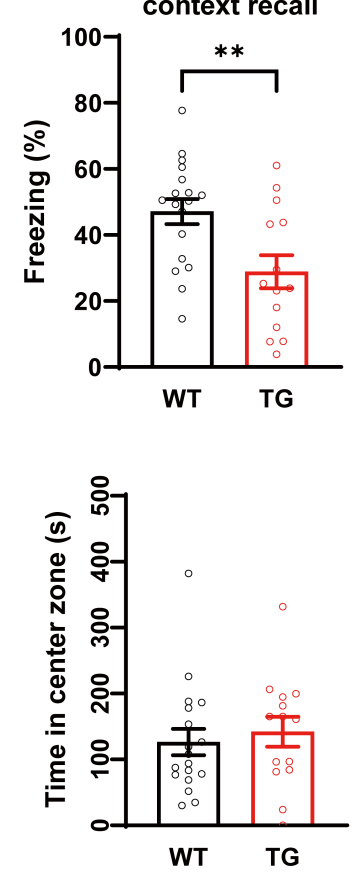

Fig. 1. Long-term memory deficit in 12-month-old AD mice. (A) Schematic representation of CFC protocol. There were distinct batches with different conditioning to context recall intertrial time interval (ITI, $2 \mathrm{~h}$ versus 4 days). (B) No difference in after-shock and context recall freezing between $2 \mathrm{~h}$ ITI WT $(\mathrm{n}=7)$ and TG $(\mathrm{n}=7)$. (C) In 4d ITI experiment, TG $(\mathrm{n}=14)$ mice show significant decrease in the context recall freezing compared to WT $(n=18)\left({ }^{* *} \mathrm{p}<0.01\right.$, Student's t-test). (D) No difference in total traveled distance and time spent in center zone during the open field test, between WT and TG of 4d ITI group.

RSC to determine the relationship between electrophysiological interplay and long-term memory dysfunction in AD mice. Slow wave sleep epochs, which are crucial for memory storage, were extracted from the LFP signals recorded in the home cage. First,

we identified SWR using hippocampus LFP and gathered LFP traces around individual SWR events (Fig. 2A). We performed spectrogram analysis of the surrounding LFPs (aligned with the SWR trough), individually z-scored, and averaged it. The spectrogram results showed a clear increase in the ripple $(100 \sim 250 \mathrm{~Hz})$ band and delta $(1 \sim 4 \mathrm{~Hz})$ band power in both hippocampus (Fig. 2B) and RSC (Fig. 2C). The peak amplitude was much greater in TG mice. Delta oscillations around SWR were significantly higher in both hippocampus (Fig. 2D; WT, 0.228 \pm 0.009 , n=13; TG, $0.285 \pm 0.010, \mathrm{n}=12 ;{ }^{* *} \mathrm{p}<0.001$, Student's t-test) and RSC (Fig. 2E; WT, $0.190 \pm 0.007, \mathrm{n}=13 ; \mathrm{TG}, 0.233 \pm 0.011, \mathrm{n}=12$; ${ }^{* *} \mathrm{p}<0.01$, Student's $\mathrm{t}$-test) in TG mice, but ripple power was increased in RSC only (Fig. 2E; WT, 0.750 $\pm 0.056, \mathrm{n}=13$; TG, $0.950 \pm 0.081, \mathrm{n}=12$; ${ }^{*} \mathrm{p}<0.05$, Student's t-test). In addition, the beta power was decreased in TG RSC during the SWR event (Fig. 2E; WT, 0.436 $\pm 0.021, \mathrm{n}=13$; TG, $0.371 \pm 0.023, \mathrm{n}=12$; ${ }^{*} \mathrm{p}<0.05$, Student's t-test).

Because of the importance of ripple band oscillation in the SWR, we further analyzed the features of the ripple wave. In TG mice, the amount of ripple power during SWR was negatively correlated with context freezing level (Fig. 2F; WT, $R^{2}=0.067, \mathrm{n}=13, \mathrm{p}=0.393$; TG, $R^{2}=0.775, \mathrm{n}=12,{ }^{* *} \mathrm{p}<0.001$; Pearson correlation). According to previous studies [23-25], RSC has ripple-like high-frequency activity, and this cortical ripple tends to coincide with SWR for successful memory consolidation. To determine the SWR and cortical ripple communication, we identified the cortical ripple in the RSC by applying the same criterion of SWR detection (Fig. 2G). The general characteristics of RSC ripple were similar in the TG mice, except for the amplitude (Table 1). The co-occurrence ratio between the SWR and nearby RSC ripple (defined as occurring within $50 \mathrm{~ms}$ from the SWR trough) and coherence was equivalent between WT and TG mice (Fig. 2H left; WT, 0.096 $\pm 0.009, \mathrm{n}=13$; TG, $0.117 \pm 0.011, n=12 ; p=0.157$, Student's t-test) (Fig. $2 \mathrm{H}$ right; WT, $0.693 \pm 0.016, n=13 ; T G, 0.720 \pm 0.018, n=12 ; \mathrm{p}=0.255$, Student's $\mathrm{t}$-test). This result implies that the increased SWR corresponding to the RSC ripple amplitude interferes with long-term memory formation, even if the quantitative and phasic ripple communications between hippocampus and RSC are intact.

\section{Increased amplitude of SWR coordinated delta oscillation in AD mice}

Unlike transient ripple events, delta waves have a much longer temporal structure. Therefore, we extracted a wider $(0.5 \mathrm{~s}$ from the SWR trough) time window for the exact analysis of SWRcoordinated delta activity. Delta-filtered LFP signals from each brain region were averaged and z-scored. Group averaged data showed that delta waves tend to show negative peaks prior to and positive peaks after the SWR center in both areas (Fig. 3A), 
A
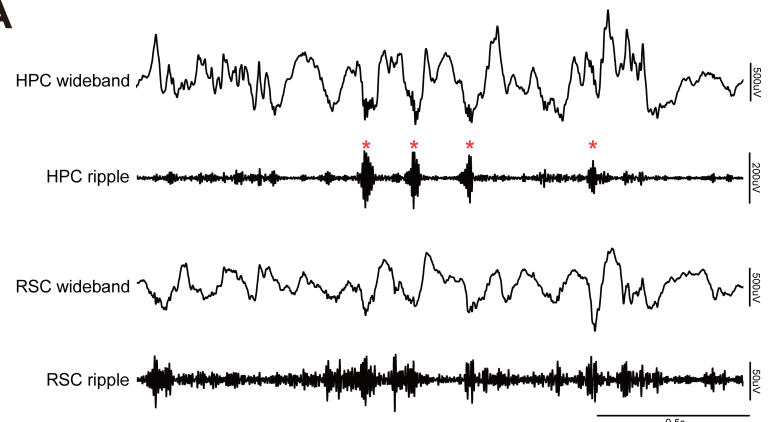

D

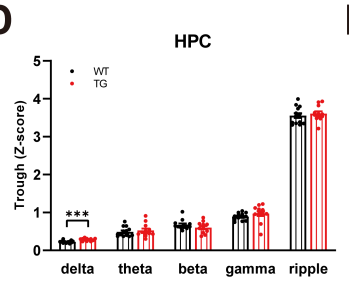

E

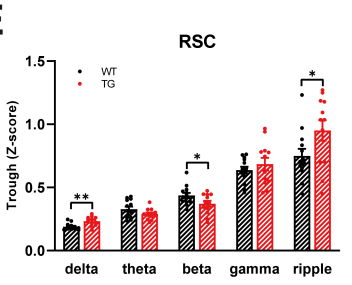

$F$
B

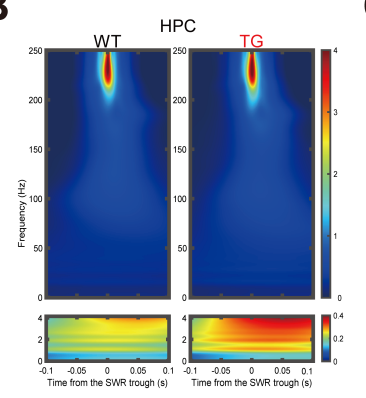

C

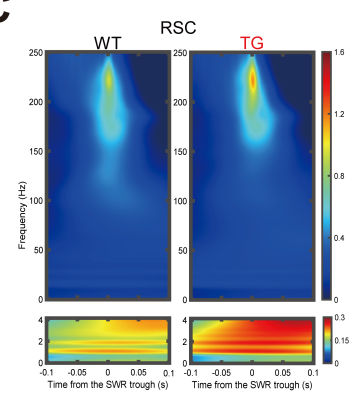

G

$\mathrm{H}$

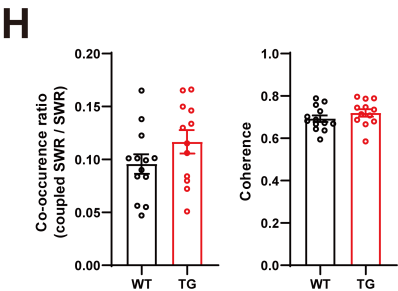

Fig. 2. Increased amplitude of SWR coordinated RSC ripple oscillation in AD mice. (A) Example traces (wideband: 1 250 Hz, ripple: 100 250 Hz) of HPC and RSC LFPs during SWS. Sharp wave ripples (SWRs) are marked on ripple filtered HPC trace (red asterisk). Note the RSC also has a prominent amplitude change in ripple band oscillation like as HPC. (B) Grand average spectrogram of HPC LFP around SWRs. Prominent ripple band power (top panels) and delta frequency power (bottom panels) changes are detected in both of WT ( $\mathrm{n}=13$ ) and TG (n=12) mice. (C) Grand average spectrogram of coordinated RSC LFP around HPC SWR events. Similar with HPC spectrogram, RSC LFPs also show high amplitude in ripple and delta oscillations around SWRs. Color bar in panel B \& C indicates the z-scored amplitude. (D) Individually quantified peak band power in HPC channel (delta: 1 4 Hz, theta: $5 \sim 10 \mathrm{~Hz}$, beta: 12 30 Hz, gamma: 31 100 Hz, ripple: 100 250 Hz). Transgenic mice show significantly increased delta power compared to WT $\left({ }^{* *} \mathrm{p}<0.001\right.$, Student's t-test). (E) Individually quantified peak band power in RSC channel. Significantly increased in ripple and delta amplitude is detected in TG mice $\left({ }^{*} \mathrm{p}<0.05\right.$ and ${ }^{* *} \mathrm{p}<0.01$ respectively, Student's t-test), together with reduced beta oscillation power. (F) Sharp wave ripple (SWR) corresponding RSC ripple amplitude is correlated with context recall freezing in TG mice (WT: $\mathrm{p}=0.393, \mathrm{TG}:{ }^{* * *} \mathrm{p}<0.001$, Pearson's correlation). (G) Example HPC LFP trace with cortical ripple in RSC (top two traces, wideband). Retrosplenial cortex (RSC) show large ripple oscillation respond to HPC SWR (bottom two traces, ripple filtered). (H) Co-occurrence rate of HPC SWR and RSC ripple (occurred within $50 \mathrm{~ms}$ from the SWR trough) is equivalent between WT and TG mice (left). Coherence between SWR and RSC ripple is not changed in TG mice (right).

similar to a previous report [24]. The general shape of delta oscillation did not change in TG mice, but the amplitude (defined as amplitude from negative to positive peak) increased (Fig. 3B HPC; WT, 0.739 $\pm 0.066, \mathrm{n}=13$; TG, 1.109 $\pm 0.083, \mathrm{n}=12$; ${ }^{* * *} \mathrm{p}<0.001$, Mann-Whitney test) (Fig. 3B RSC; WT, $0.883 \pm 0.073$, n=13; TG, $1.174 \pm 0.051, \mathrm{n}=12 ;{ }^{* *} \mathrm{p}<0.01$, Student's $\mathrm{t}$-test). The duration between peak points was also expanded in RSC in TG mice (Fig. 3C; WT, $0.181 \pm 0.002, n=13$; TG, $0.191 \pm 0.002, n=12$; ${ }^{*} \mathrm{p}<0.05$, Student's $\mathrm{t}$-test). The higher delta amplitudes in TG mice were negatively correlated with the recall freezing time, reminiscent of previous ripple wave results (Fig. 3D left; WT, $R^{2}=0.343, \mathrm{n}=13,{ }^{*} \mathrm{p}<0.05$; TG, $R^{2}=0.276, \mathrm{n}=12, \mathrm{p}=0.079 ;$ Pearson correlation; Fig. 3D middle; WT, $R^{2}=0.030, \mathrm{n}=13, \mathrm{p}=0.573$; TG, $R^{2}=0.499, \mathrm{n}=12,{ }^{*} \mathrm{p}<0.05$; Pearson correlation). The hippocampus delta amplitude showed a correlation with freezing time in the TG mice group, though not statistically significant. Further, we investigated the link between the delta powers of two brain regions. Both groups showed significant correlations in regional delta amplitudes (Fig. 3D right; $\mathrm{WT}, R^{2}=0.452$, $\mathrm{n}=13,{ }^{*} \mathrm{p}<0.05$; TG, $R^{2}=0.569, \mathrm{n}=12,{ }^{* *} \mathrm{p}<0.01$; Pearson correlation), indicating a mutual effect in delta waves.

\section{Reduced difference of SWR coordinated delta phases in $A D$ mice}

High-frequency oscillations are relatively temporary events compared to low-frequency oscillations. Thus, fast waves can be located within a particular phase of slower waves, and this process is known to lead to the integration of neuronal population $[15,16]$. To investigate the phasic relationship between the SWR and coordinated delta oscillation, we analyzed the hippocampus and RSC delta phases corresponding to individual SWR outbreak points (Fig. 4A). Most SWRs were nested within a similar phase of HPC and RSC, described as high probability along the diagonal line (Fig. $4 \mathrm{~B})$. There was no difference in the average phase of each brain area between the WT and TG mice. However, the paired relationship of the delta phase between hippocampus and RSC was different. (Fig. 4C; WT, $\mathrm{n}=13,{ }^{\sharp \neq \# *} \mathrm{p}<0.0001$; TG, $\mathrm{n}=12, \mathrm{p}=0.109$, Paired ttest). This result can be represented as a decreased phase difference between the two regions in the TG (Fig. 4D; WT, 26.901 \pm 4.014 , 
Table 1. Characteristics of SWS sleep oscillation events

\begin{tabular}{rlrrr}
\hline \multicolumn{2}{c}{ Sleep oscillations } & \multicolumn{1}{c}{ WT } & \multicolumn{1}{c}{ TG } & p-value \\
\hline \multirow{2}{*}{ SWR } & Amplitude & $3.556 \pm 0.064$ & $3.609 \pm 0.058$ & 0.543 \\
& Occurrence & $0.548 \pm 0.025$ & $0.412 \pm 0.027$ & $<0.001^{* * *}$ \\
& Duration & $25.718 \pm 0.532$ & $24.258 \pm 0.558$ & 0.071 \\
\multirow{3}{*}{ RSC ripple } & Frequency & $141.764 \pm 0.358$ & $141.038 \pm 0.943$ & 0.466 \\
& Amplitude & $2.992 \pm 0.035$ & $3.521 \pm 0.065$ & $<0.001^{* * *}$ \\
& Occurrence & $0.295 \pm 0.017$ & $0.346 \pm 0.029$ & 0.134 \\
& Duration & $20.325 \pm 0.126$ & $20.035 \pm 0.124$ & 0.115 \\
RSC SPD & Frequency & $150.342 \pm 0.787$ & $148.571 \pm 0.622$ & 0.094 \\
& Amplitude & $3.363 \pm 0.043$ & $3.361 \pm 0.040$ & 0.975 \\
& Occurrence & $0.129 \pm 0.002$ & $0.125 \pm 0.003$ & 0.238 \\
RSC SO & Duration & $0.856 \pm 0.008$ & $0.866 \pm 0.010$ & 0.450 \\
& Frequency & $9.969 \pm 0.029$ & $9.992 \pm 0.027$ & 0.573 \\
& Amplitude & $3.809 \pm 0.018$ & $3.795 \pm 0.012$ & 0.547 \\
& Occurrence & $0.546 \pm 0.014$ & $0.531 \pm 0.011$ & 0.387 \\
\end{tabular}

Amplitude (Z-score), occurrence (Events/s), duration (ms : SWR and RSC ripple, s: SPD and SO), frequency (Hz).

$\mathrm{p}$-value indicates the significance of WT and TG comparisons. Significant results are represented in bold type ${ }^{* * *} \mathrm{p}<0.001$, Student's t-test).

$\mathrm{n}=13$; TG, $9.051 \pm 5.187, \mathrm{n}=12$; ${ }^{*} \mathrm{p}<0.05$, Student's t-test). The amount of the delta phase difference was positively correlated with the context recall freezing (Fig. $4 \mathrm{E} ; \mathrm{WT}, R^{2}=0.499, \mathrm{n}=13,{ }^{* *} \mathrm{p}<0.01$; $\mathrm{TG}, R^{2}=0.352, \mathrm{n}=12,{ }^{*} \mathrm{p}<0.05$; Pearson correlation), indicating that delta communication around SWR events might be a critical factor for long-term memory formation.

\section{Altered sleep oscillation coupling between HPC and RSC in AD mice}

Along with SWR in HPC, SO and SPD are critical SWS oscillations detected in the cortices. The temporal coupling between these events has been regarded as a proxy for memory consolidation. The general features of SO and SPD were similar despite the reduced SWR occurrence in TG mice (Table 1). Temporal coupling can be divided into serial events implying the sequential occurrence of SWR-SO-SPD and events with overlap, indicating an outbreak of SWR within the SO or SPD period [16] (Fig. $5 \mathrm{~A}$; events in serial: green box; event with overlap: magenta box). In the case of coupled SWR and SO, the occurrence ratio did not change, but serial SO and SPD events were decreased in the TG mice (Fig. 5B SWR $\rightarrow$ SO; WT, 0.208 \pm 0.013 , n=13; TG, $0.204 \pm 0.014, \mathrm{n}=12 ; \mathrm{p}=0.837$, Student's t-test) (Fig. $5 \mathrm{~B} \mathrm{SO} \rightarrow \mathrm{SPD}$; WT, $0.194 \pm 0.004, \mathrm{n}=13$; TG, $0.178 \pm 0.005, \mathrm{n}=12$; ${ }^{*} \mathrm{p}<0.05$, Student's t-test). The triple events ratio was conserved, which seems to be due to the unchanged SWR $\rightarrow$ SO ratio (Fig. $5 \mathrm{~B} S W R \rightarrow S O \rightarrow S P D$; WT, $0.046 \pm 0.003, \mathrm{n}=13$; TG, $0.039 \pm 0.002, \mathrm{n}=12 ; \mathrm{p}=0.081$, Student's $\mathrm{t}$-test). We then analyzed the event overlapping ratio by comparing the total number of SWRs in each mouse. Trends were significant but in different directions (Fig. 5C SWR within SO; WT, $0.207 \pm 0.010, \mathrm{n}=13 ; \mathrm{TG}, 0.274 \pm 0.009, \mathrm{n}=12 ;{ }^{* * *} \mathrm{p}<0.0001$, Student's t-test) (Fig. 5C SWR within SPD; WT, 0.214 $\pm 0.008, \mathrm{n}=13$; TG, $0.183 \pm 0.007, n=12 ;{ }^{* *} \mathrm{p}<0.01$, Student's $\mathrm{t}$-test). None of the above features correlated with freezing level in context recall, although they were different from those of the WT mice (Fig. 5D).

\section{DISCUSSION}

We investigated the relationship between memory performance and SWR corresponding to RSC activity in an AD mouse model during sleep. First, AD mice had a long-term memory deficit, as expected. In contrast to previous studies [12,13], the exact timing of the symptoms were distinct. This difference can be explained by the fact that the onset age of memory deficit varies with previous reports [12, 13, 32-34]. The details of the CFC protocol and the conditions of the behavior setup could also lead to this disparity. Despite this difference, it is quite reproducible, at least in the AD mouse model.

AD mice showed increased amplitude in the SWR-coordinated RSC oscillation, ripple, and delta band. The amplitude of ripple and delta oscillations were negatively correlated with memory performance, specifically in the TG group. Due to the unaltered SWR amplitude in TG mice, elevated ripple amplitude might be a local issue within the RSC. The co-occurrence ratio and coherence between SWR and RSC ripple were intact, implying that the problem might be in the reception unit rather than ripple communication between the hippocampus and RSC. Increased ripple power might indicate increased excitability in the RSC. Increased excitability in an $\mathrm{AD}$ mouse model is a well-known characteristic of seizures in various models [35]. In particular, unexpected neural excitation during SWR could hinder the actual component of cognitive 
A
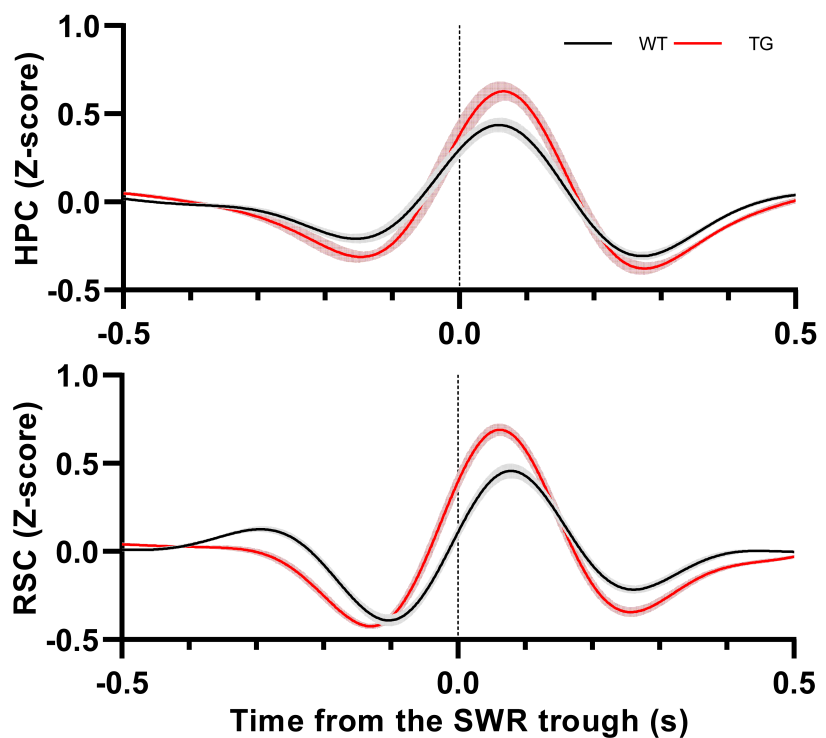

D

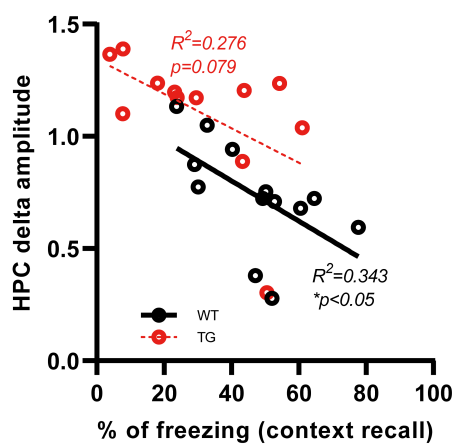

B

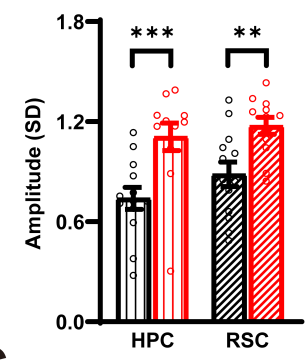

C

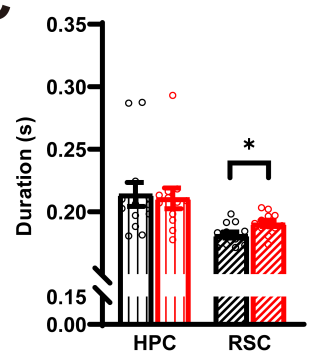

Fig. 3. Increased amplitude of SWR coordinated RSC delta oscillation in AD mice. (A) Grand average of raw delta trace around SWRs. Shaded area indicates SEM of averaged delta wave. Note that, both HPC (top panel) and RSC (bottom panel) show the negative and positive peaks before and after the SWR trough respectively. (B) Amplitude (negative peak to positive peak) of SWR related delta oscillation is higher in TG mice in both HPC and RSC $\left({ }^{* *} \mathrm{p}<0.001\right.$, Mann-Whitney test, ${ }^{* *} \mathrm{p}<0.05$, Student's t-test). (C) Duration (from negative peak to positive peak) of delta wave is significantly increased at the RSC of TG mice ${ }^{* *} \mathrm{p}<0.05$, Student's t-test). (D) HPC (left panel) show negative correlation with freezing level in context recall session, although TG only shows marginal $\mathrm{p}$-value (WT: ${ }^{*} \mathrm{p}<0.05$, TG: $\mathrm{p}=0.08$, Pearson's correlation). Only TG mice showed significant correlation between RSC delta power (middle panel) and recall freezing level (WT: $\mathrm{p}=0.573$, TG: ${ }^{*} \mathrm{p}<0.05$, Pearson's correlation). Delta amplitude of HPC and RSC closely associated regardless of genotype (right panel, WT: ${ }^{*} \mathrm{p}<0.05, \mathrm{TG}:{ }^{* *} \mathrm{p}<0.01$, Pearson's correlation).

information, called a memory engram. Unnecessary action potentials act as noise that obstructs selective synaptic potentiation [36]. This excitatory-inhibitory imbalance could be the result of selective loss of inhibitory neuronal function [37-39]. Therefore, the increased ripple amplitude in the RSC might be a reflection of these underlying mechanisms.

Recent study measured the directional coupling between hippocampus and several cortical areas in other type of APP/PS1 mouse model [26]. The study identified the switched direction of information flow from RSC (including posterior cingulate cortex) to frontal cortex in SWR dependent ripple oscillation. Also, they found the reduced gamma band $(20 \sim 60 \mathrm{~Hz})$ coupling between hippocampus CA1 and RSC in AD mice. This study implies the disrupted interplay between hippocampus and RSC could be substituted by other cortical areas. Although we did not measure the rearrangement of communication between hippocampus and other cortical areas, RSC seems to be the core area showing the altered relationship with hippocampus in $\mathrm{AD}$ mouse model.

Unlike ripple waves, increased nearby delta oscillations were also detected in the hippocampus. Amplitudes of delta waves were closely correlated with each brain region regardless of genotype. The SWR-coordinated delta oscillation also showed a lead and lag relationship between the hippocampus and RSC; however, TG mice showed a high synchrony between them, as inferred by the 
A

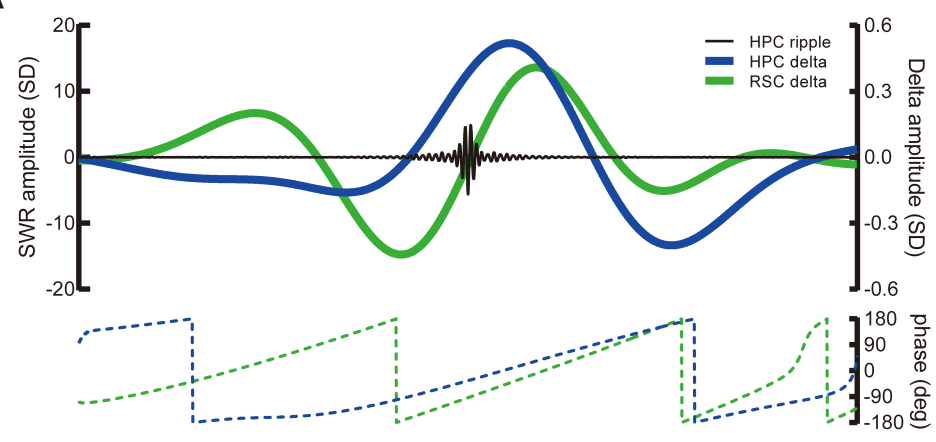

B
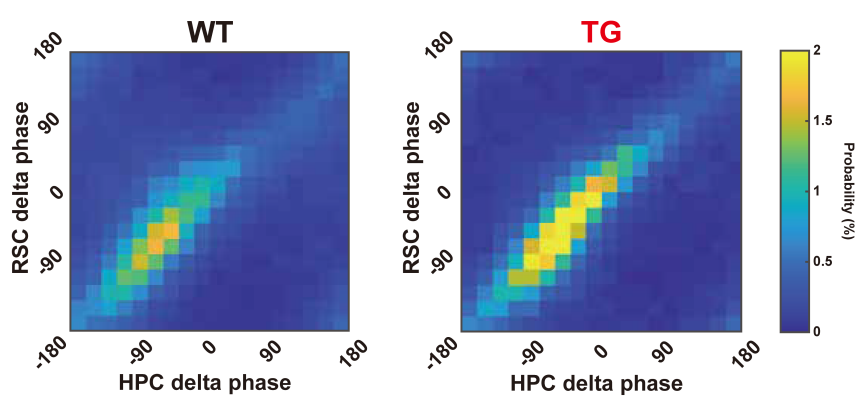

C

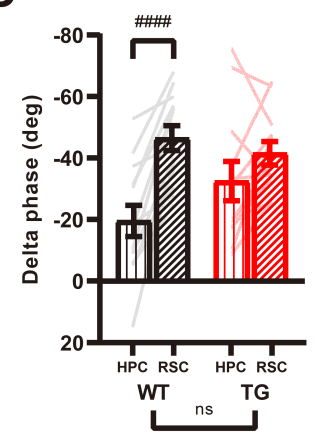

D

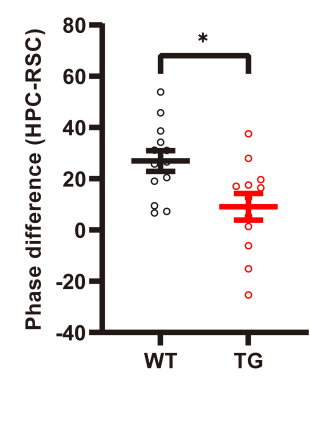

E

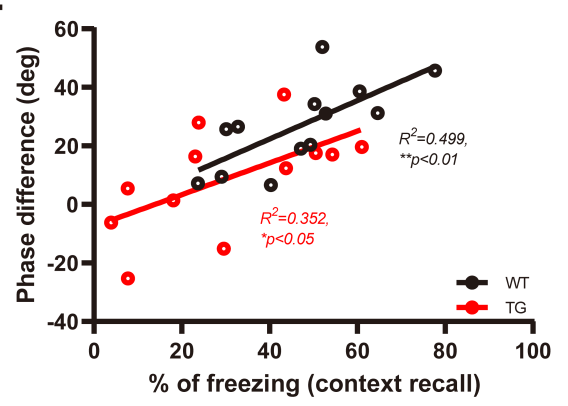

Fig. 4. Reduced difference of SWR coordinated delta phases in AD mice. (A) Example of SWR trace (black) along with delta oscillation from HPC (blue) and RSC (green). Corresponding delta phase change is represented in below. (B) Group averaged SWR nesting delta phase in scaled matrix. The phase location of each SWR is analyzed and divided by total SWR number of each mouse. (C) Median delta phase of HPC and RSC in individual. There is no group difference in regional phase, but the regional relationship is changed (WT HPC-RSC, ${ }^{\# \# \# ~} \mathrm{p}<0.0001$, paired t-test). (D) Delta phase difference between HPC and RSC is decreased in TG mice ${ }^{*} \mathrm{p}<0.05$, Student's t-test). (E) Phase difference is positively correlated with context recall freezing level (WT: ${ }^{*} \mathrm{p}<0.05, \mathrm{TG}:{ }^{* *} \mathrm{p}<0.01$, Pearson's correlation).

decreased phase difference between regions. The results indicate the importance of delta amplitude as well as the delta phasic interplay in context memory recall. A previous report found that the RSC negative and positive delta peaks around SWR represent up and down states, respectively [24]. Although the exact role of RSC population activity occurring around SWR remains unknown, it is plausible that the above results connote altered neural activation around SWR.

Previous study identified that the changed SWR-SO and spindleSO coupling between hippocampus and parietal cortex in $\mathrm{AD}$ mouse model of tauopathy and amyloidosis [40]. This paper suggests the impairment in hippocampal-cortical coordination of memory replay events, however it is not sure whether the altered relationship is the general characteristic across the cortical area and regardless of $\mathrm{AD}$ mouse model of amyloidosis. Considering the difference in AD model, stage of disease and LFP extraction time point, it is difficult to direct comparison. However, still our results are in the same line in terms of the alteration in spindle-SO serial coupling.

This study covered only the characteristics of the field potentials in the hippocampus and RSC during SWS. Therefore, we could not suggest the source of increased amplitude or determine the correlation between increased amplitude and actual changes in neuronal population activity, in terms of memory engram and replay. Utilizing the simultaneous recording of individual neuronal activity, classifying between type (excitatory or inhibitory) and function (encoded memory engram or unrelated population), and identifying alterations in the neuronal group would be necessary for a better understanding of the current results. Although the role of REMS has been relatively overlooked, recent study identified that Granger causality between the hippocampus and RSC is important factor in successful memory consolidation during REMS [41]. This study raises the possibility of disrupted hippocampus and RSC coupling during REMS, which is not analyzed in our study. Further investigation is needed to expand our knowledge about fundamental sleep oscillation communication between two regions and episodic memory dysfunction under $\mathrm{AD}$ pathology.

\section{ACKNOWLEDGEMENTS}

This research was supported by the Basic Research Lab (BRL) Program (NRF-2020R1A4A1018714) funded by the Korean 
A

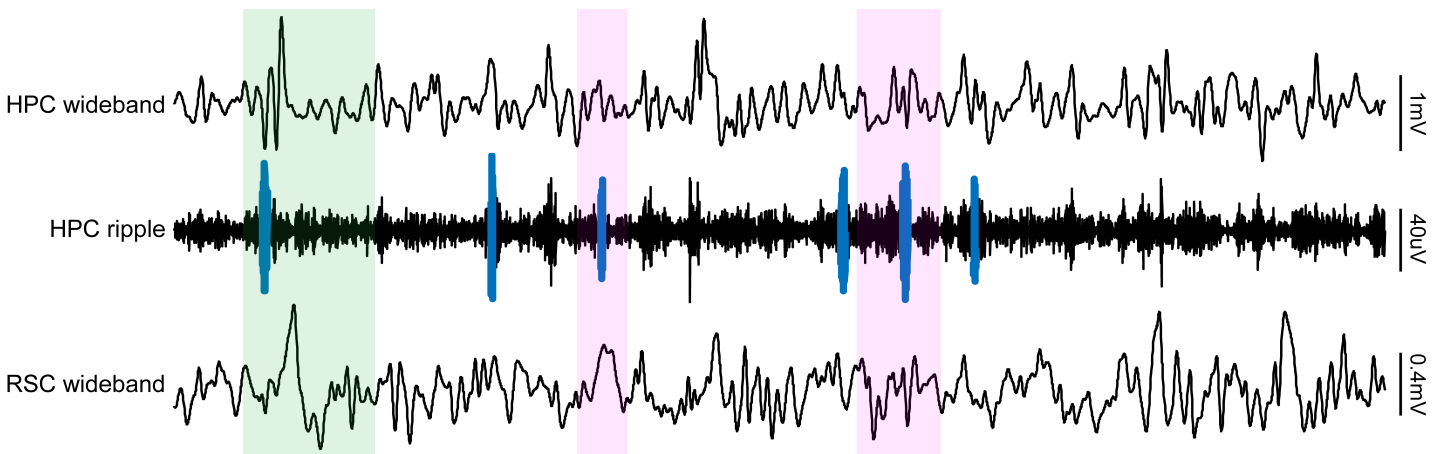

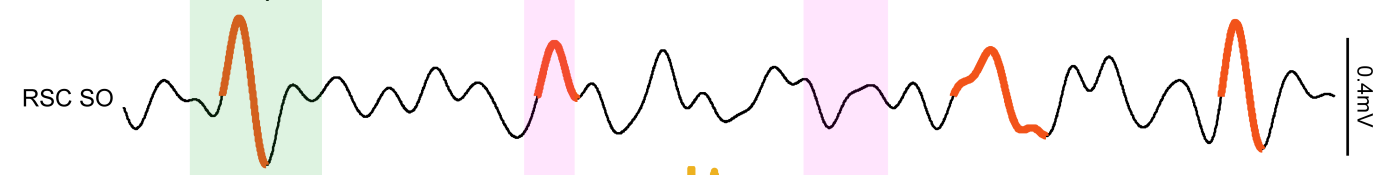

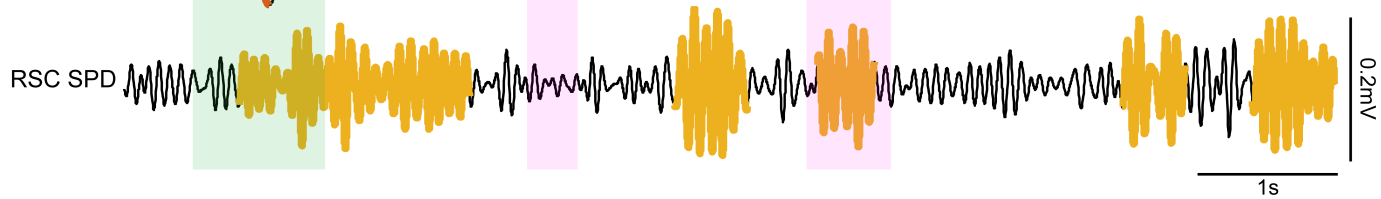

B
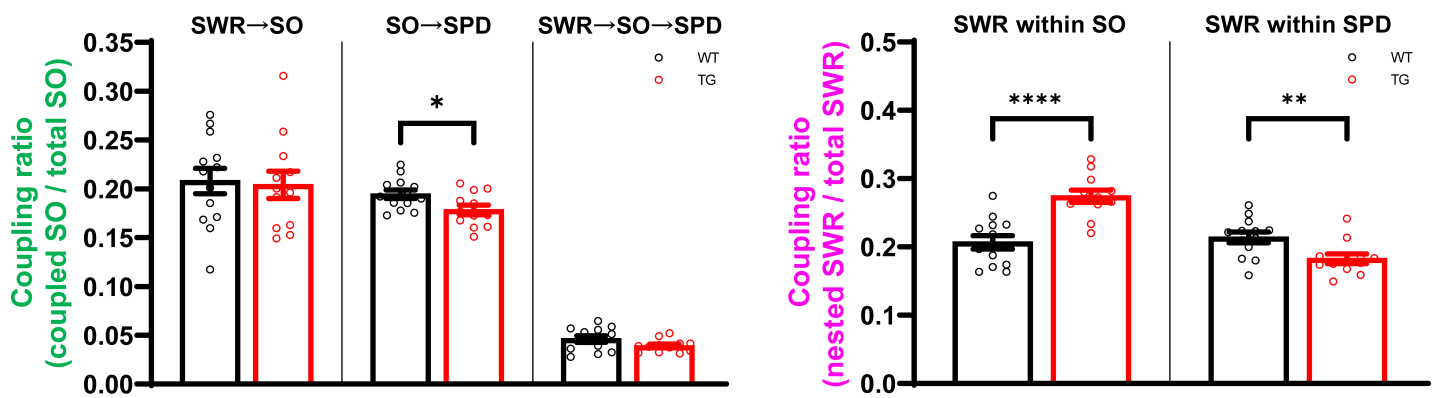

D
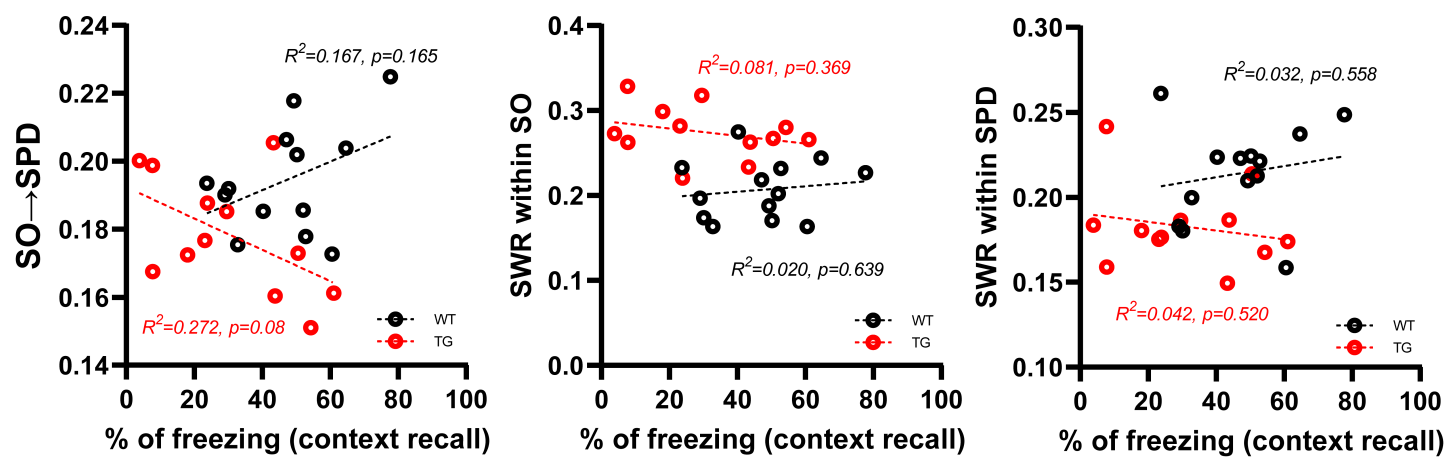

Fig. 5. Altered sleep oscillation coupling between HPC and RSC in AD mice. (A) Example traces of HPC and RSC during SWS. Sharp wave ripple is marked on HPC ripple band (100 250 Hz) filtered trace with blue line. Cortical slow oscillation (SO) and spindle (SPD) events are labeled with red and yellow line respectively on filtered RSC channel (RSC SO : RSC $0.5 \sim 4 \mathrm{~Hz}$ filtered trace, RSC SPD : RSC 7 15 Hz filtered trace). Note the serial event of SWR-SO-SPD (green box) and co-occurrence of SWR within SO and SPD (magenta box). (B) Sequential appearance of SWR-SO and SWR-SO-SPD is unchanged, but SO-SPD pair event is reduced in TG mice $\left({ }^{*} \mathrm{p}<0.05\right.$, Student's t-test). (C) Co-occurrence ratio of SWR-SO and SWR-SPD is altered in TG mice $\left({ }^{* *} \mathrm{p}<0.01,{ }^{* *} \mathrm{p}<0.0001\right.$, Student's t-test). (D) No correlation between changed HPC-RSC sleep oscillation coupling properties and freezing level in context recall. 
Government (MSIP) through the National Research Foundation (NRF) and by the Technology Innovation Program (20012464) funded by the Ministry of Trade, Industry \& Energy (MOTIE, Korea).

\section{REFERENCES}

1. Knopman DS, Amieva H, Petersen RC, Chételat G, Holtzman DM, Hyman BT, Nixon RA, Jones DT (2021) Alzheimer disease. Nat Rev Dis Primers 7:33.

2. Ballard C, Gauthier S, Corbett A, Brayne C, Aarsland D, Jones E (2011) Alzheimer's disease. Lancet 377:1019-1031.

3. Weintraub S, Wicklund AH, Salmon DP (2012) The neuropsychological profile of Alzheimer disease. Cold Spring Harb Perspect Med 2:a006171.

4. Pause BM, Zlomuzica A, Kinugawa K, Mariani J, Pietrowsky R, Dere E (2013) Perspectives on episodic-like and episodic memory. Front Behav Neurosci 7:33.

5. Tromp D, Dufour A, Lithfous S, Pebayle T, Després O (2015) Episodic memory in normal aging and Alzheimer disease: insights from imaging and behavioral studies. Ageing Res Rev 24(Pt B):232-262.

6. Peña-Casanova J, Sánchez-Benavides G, de Sola S, ManeroBorrás RM, Casals-Coll M (2012) Neuropsychology of Alzheimer's disease. Arch Med Res 43:686-693.

7. Perri R, Serra L, Carlesimo GA, Caltagirone C; Early Diagnosis Group of the Italian Interdisciplinary Network on Alzheimer's Disease (2007) Amnestic mild cognitive impairment: difference of memory profile in subjects who converted or did not convert to Alzheimer's disease. Neuropsychology 21:549-558.

8. Hart RP, Kwentus JA, Harkins SW, Taylor JR (1988) Rate of forgetting in mild Alzheimer's-type dementia. Brain Cogn 7:31-38.

9. Hodges JR, Salmon DP, Butters N (1990) Differential impairment of semantic and episodic memory in Alzheimer's and Huntington's diseases: a controlled prospective study. J Neurol Neurosurg Psychiatry 53:1089-1095.

10. Moulin CJ, James N, Freeman JE, Jones RW (2004) Deficient acquisition and consolidation: intertrial free recall performance in Alzheimer's disease and mild cognitive impairment. J Clin Exp Neuropsychol 26:1-10.

11. Moss MB, Albert MS, Butters N, Payne M (1986) Differential patterns of memory loss among patients with Alzheimer's disease, Huntington's disease, and alcoholic Korsakoff's syndrome. Arch Neurol 43:239-246.

12. Kilgore M, Miller CA, Fass DM, Hennig KM, Haggarty SJ,
Sweatt JD, Rumbaugh G (2010) Inhibitors of class 1 histone deacetylases reverse contextual memory deficits in a mouse model of Alzheimer's disease. Neuropsychopharmacology 35:870-880.

13. Roy DS, Arons A, Mitchell TI, Pignatelli M, Ryan TJ, Tonegawa $S$ (2016) Memory retrieval by activating engram cells in mouse models of early Alzheimer's disease. Nature 531:508512.

14. Walker MP (2009) The role of slow wave sleep in memory processing. J Clin Sleep Med 5(2 Suppl):S20-S26.

15. Sirota A, Csicsvari J, Buhl D, Buzsáki G (2003) Communication between neocortex and hippocampus during sleep in rodents. Proc Natl Acad Sci U S A 100:2065-2069.

16. Marshall L, Cross N, Binder S, Dang-Vu TT (2020) Brain rhythms during sleep and memory consolidation: neurobiological insights. Physiology (Bethesda) 35:4-15.

17. Helfrich RF, Lendner JD, Mander BA, Guillen H, Paff M, Mnatsakanyan L, Vadera S, Walker MP, Lin JJ, Knight RT (2019) Bidirectional prefrontal-hippocampal dynamics organize information transfer during sleep in humans. Nat Commun 10:3572.

18. Ji D, Wilson MA (2007) Coordinated memory replay in the visual cortex and hippocampus during sleep. Nat Neurosci 10:100-107.

19. Wang G, Grone B, Colas D, Appelbaum L, Mourrain P (2011) Synaptic plasticity in sleep: learning, homeostasis and disease. Trends Neurosci 34:452-463.

20. Joo HR, Frank LM (2018) The hippocampal sharp wave-ripple in memory retrieval for immediate use and consolidation. Nat Rev Neurosci 19:744-757.

21. Buzsáki G (2015) Hippocampal sharp wave-ripple: a cognitive biomarker for episodic memory and planning. Hippocampus 25:1073-1188.

22. Todorova R, Zugaro M (2020) Hippocampal ripples as a mode of communication with cortical and subcortical areas. Hippocampus 30:39-49.

23. Nitzan N, McKenzie S, Beed P, English DF, Oldani S, Tukker JJ, Buzsáki G, Schmitz D (2020) Propagation of hippocampal ripples to the neocortex by way of a subiculum-retrosplenial pathway. Nat Commun 11:1947.

24. Opalka AN, Huang WQ, Liu J, Liang H, Wang DV (2020) Hippocampal ripple coordinates retrosplenial inhibitory neurons during slow-wave sleep. Cell Rep 30:432-441.e3.

25. Khodagholy D, Gelinas JN, Buzsáki G (2017) Learningenhanced coupling between ripple oscillations in association cortices and hippocampus. Science 358:369-372.

26. Jura B, Młoźniak D, Goszczyńska H, Blinowska K, Biendon N, 
Macrez N, Meyrand P, Bem T (2020) Reconfiguration of the cortical-hippocampal interaction may compensate for SharpWave Ripple deficits in APP/PS1 mice and support spatial memory formation. PLoS One 15:e0243767.

27. Todd TP, Bucci DJ (2015) Retrosplenial cortex and long-term memory: molecules to behavior. Neural Plast 2015:414173.

28. Yamawaki N, Li X, Lambot L, Ren LY, Radulovic J, Shepherd GMG (2019) Long-range inhibitory intersection of a retrosplenial thalamocortical circuit by apical tuft-targeting CA1 neurons. Nat Neurosci 22:618-626.

29. Maingret N, Girardeau G, Todorova R, Goutierre M, Zugaro M (2016) Hippocampo-cortical coupling mediates memory consolidation during sleep. Nat Neurosci 19:959-964.

30. Latchoumane CV, Ngo HV, Born J, Shin HS (2017) Thalamic spindles promote memory formation during sleep through triple phase-locking of cortical, thalamic, and hippocampal rhythms. Neuron 95:424-435.e6.

31. Niethard N, Ngo HV, Ehrlich I, Born J (2018) Cortical circuit activity underlying sleep slow oscillations and spindles. Proc Natl Acad Sci U S A 115:E9220-E9229.

32. Végh MJ, Heldring CM, Kamphuis W, Hijazi S, Timmerman AJ, Li KW, van Nierop P, Mansvelder HD, Hol EM, Smit AB, van Kesteren RE (2014) Reducing hippocampal extracellular matrix reverses early memory deficits in a mouse model of Alzheimer's disease. Acta Neuropathol Commun 2:76.

33. Lesuis SL, van Hoek BACE, Lucassen PJ, Krugers HJ (2017) Early postnatal handling reduces hippocampal amyloid plaque formation and enhances cognitive performance in APPswe/PS1dE9 mice at middle age. Neurobiol Learn Mem 144:27-35.

34. O'Leary TP, Hussin AT, Gunn RK, Brown RE (2018) Locomotor activity, emotionality, sensori-motor gating, learning and memory in the APPswe/PS1dE9 mouse model of Alzheimer's disease. Brain Res Bull 140:347-354.

35. Scharfman HE (2012) Alzheimer's disease and epilepsy: insight from animal models. Future Neurol 7:177-192.

36. Poll S, Mittag M, Musacchio F, Justus LC, Giovannetti EA, Steffen J, Wagner J, Zohren L, Schoch S, Schmidt B, Jackson WS, Ehninger D, Fuhrmann M (2020) Memory trace interference impairs recall in a mouse model of Alzheimer's disease. Nat Neurosci 23:952-958.

37. Xu Y, Zhao M, Han Y, Zhang H (2020) GABAergic inhibitory interneuron deficits in Alzheimer's disease: implications for treatment. Front Neurosci 14:660.

38. Shimojo M, Takuwa H, Takado Y, Tokunaga M, Tsukamoto S, Minatohara K, Ono M, Seki C, Maeda J, Urushihata T, Minamihisamatsu T, Aoki I, Kawamura K, Zhang MR, Suhara T, Sahara N, Higuchi M (2020) Selective disruption of inhibitory synapses leading to neuronal hyperexcitability at an early stage of tau pathogenesis in a mouse model. J Neurosci 40:3491-3501.

39. Hijazi S, Heistek TS, Scheltens P, Neumann U, Shimshek DR, Mansvelder HD, Smit AB, van Kesteren RE (2020) Early restoration of parvalbumin interneuron activity prevents memory loss and network hyperexcitability in a mouse model of Alzheimer's disease. Mol Psychiatry 25:3380-3398.

40. Benthem SD, Skelin I, Moseley SC, Stimmell AC, Dixon JR, Melilli AS, Molina L, McNaughton BL, Wilber AA (2020) Impaired hippocampal-cortical interactions during sleep in a mouse model of Alzheimer's disease. Curr Biol 30:2588-2601. e5.

41. de Almeida-Filho DG, Koike BDV, Billwiller F, Farias KS, de Sales IRP, Luppi PH, Ribeiro S, Queiroz CM (2021) Hippocampus-retrosplenial cortex interaction is increased during phasic REM and contributes to memory consolidation. Sci Rep 11:13078. 